\title{
Ciudad, territorio y exclusión social. Las políticas de recualificación urbana en la ciudad de Buenos Aires
}

Fernando Díaz Orueta María Luisa Lourés

Universidad de Alicante

Carla Rodríguez

Verónica Devalle

Universidad de Buenos Aires

RESUMEN

El análisis de la exclusión social en las ciudades se dirige, con frecuencia, hacia áreas muy delimitadas donde se concentran las situaciones más extremas de malestar urbano. De hecho, cuando se dan, es en esas zonas donde confluyen los principales planes y programas de intervención implementados desde la Administración. Sin embargo, la exclusión no presenta siempre una expresión territorial tan clara. Así, la agudización de los procesos de fragmentación social no se traduce, necesariamente, en la formación de espacios fuertemente segregados. En el artículo se analiza la evolución reciente de la ciudad de Buenos Aires y, más concretamente, de dos barrios de la zona sur: San Telmo y La Boca. En ambos casos existen diversos proyectos de recualificación urbana, ligados al desarrollo de la economía cultural. Sin embargo, las experiencias estudiadas, a pesar de producirse de manera simultánea y en un contexto de crisis estructural de la economía argentina, presentan particularidades muy importantes. Se trata de dos formaciones socioespaciales diferentes, con distintos grados de articulación social y, por lo tanto, construyendo respuestas sociales específicas a los procesos de reestructuración urbana.

Palabras clave: Ciudades, Exclusión Social, Política Local, Argentina. 


\section{INTRODUCCIÓN ${ }^{1}$}

Entender la exclusión como un proceso social de carácter multidimensional implica introducir en el análisis de una manera preferente la variable espacial. El territorio es percibido, entonces, no como el soporte imprescindible sobre el cual identificar las situaciones de pobreza y vulnerabilidad, sino como un factor activo, fundamental para comprender en toda su complejidad la génesis y la evolución de los procesos de exclusión social. Procesos que, en las ciudades, deben ser entendidos vinculados a las nuevas dinámicas de diferenciación socioespacial.

Partiendo de esta premisa, en las páginas siguientes se realiza una aproximación al debate sobre la exclusión, la ciudad y el territorio, sistematizando una serie de aportaciones que permiten avanzar hacia la concreción de un marco de análisis. En este primer apartado del artículo se analizan los nuevos procesos de segmentación social y espacial en el marco de las transformaciones experimentadas por las ciudades a lo largo de las dos últimas décadas. La profundización de las tendencias favorecedoras de la segregación socioespacial y la exclusión se ha visto reforzada por la puesta en marcha de políticas urbanas orientadas, primordialmente, al fortalecimiento de las ciudades como actores competitivos en el escenario mundial de la globalización económica. Numerosos sectores sociales, componentes de un tejido social roto en fragmentos, encuentran enormes dificultades para hacerse con un lugar propio, quedando relegados a los márgenes de la ciudad. La fragmentación social es también fragmentación de la trama socioorganizativa y, por tanto, imposibilidad de incorporación a la ciudad como sujetos políticos. Esta dificultad se ve confirmada cotidianamente con la sustitución de las políticas universales de bienestar social por las acciones focalizadas de lucha contra la pobreza.

En la segunda parte del artículo se estudia la evolución de una gran ciudad latinoamericana, Buenos Aires, a la luz de los planteamientos anteriores. El análisis de los barrios de San Telmo y La Boca, ubicados ambos en la zona sur de la ciudad y sometidos a políticas de intervención urbana de distinta naturaleza, permite reflexionar sobre la vinculación entre los procesos de exclusión social, el espacio y los intentos de articular respuestas desde el campo popular a los problemas más acuciantes de naturaleza urbana, singularmente el habitacional. La investigación permite identificar tensiones urbanas y

\footnotetext{
1 Este artículo recoge los resultados de la investigación Exclusión social y renovación urbana: nuevas estrategias de desarroIlo local. Buenos Aires-Argentina, financiada por el Programa de Cooperación Científica con Iberoamérica del Ministerio de Educación, Cultura y Deportes de España (convocatorias 2000, 2001 y 2002). El proyecto, cuyo director es Fernando Díaz Orueta, se realiza de manera conjunta entre un equipo de investigadores del Departamento de Sociología II de la Universidad de Alicante (España) y otro del Instituto de Investigaciones Sociales Gino Germani de la Universidad de Buenos Aires (Argentina).
} 
tendencias socioeconómicas y espaciales muy similares a las detectadas en otras grandes ciudades pero, a la vez, también muestra diversas particularidades derivadas, en gran medida, de la naturaleza específica del modelo económico desplegado en Argentina en los últimos veinticinco años.

A partir de esta experiencia, se realizan una serie de consideraciones sobre los procesos de exclusión y segmentación social detectados en la ciudad. Buenos Aires, mayoritariamente, ha orientado su agenda urbana hacia la priorización de políticas y actuaciones que no sólo no han afrontado de manera primordial la cuestión de la exclusión, sino que la han reforzado de forma sistemática.

\section{REESTRUCTURACIÓN ECONÓMICA, TERRITORIO Y EXCLUSIÓN SOCIAL}

A partir de mediados de los años setenta se inicia una profunda reestructuración económica de amplias repercusiones sociales, políticas y culturales. Diversos autores han analizado las transformaciones producidas, situándolas dentro del paso de un modelo de acumulación fordista a otro flexible (D. Harvey, 1989; A. Leborgne y A. Lipietz, 1994, etc.). En esta nueva etapa, las ciudades, y en especial aquellas integradas dentro del circuito mundial de ciudades globales, se constituyen en escenarios esenciales de las nuevas actividades económicas, a la vez que concentran y permiten visualizar parte de los fenómenos producidos por el nuevo modelo. S. Sassen (1991) señala cómo la concentración de empresas de servicios avanzados que ocupan a trabajadores altamente cualificados y su contraparte de trabajadores ligados a la prestación de los servicios demandados por estos sectores, y que se desenvuelven frecuentemente en el ámbito de la economía sumergida, tiene implicaciones concretas sobre la jerarquización del espacio urbano.

Desde diferentes perspectivas, numerosos investigadores han venido estudiando en profundidad las características del nuevo escenario emergente: el impacto provocado por la desindustrialización en las grandes áreas urbanas, la revolución tecnológica y las transformaciones que ésta lleva aparejada en amplias esferas de la vida social y económica (P. Hall, 1988; M. Castells, 1997-98, entre otros); las transformaciones de los hábitos de consumo respecto a la etapa fordista (S. Zukin, 1995); los cambios operados en la composición familiar y el papel central de la mujer en todo este proceso; las transformaciones en los sistemas de regulación del empleo (R. Boyer, 1988); la crisis del Estado de Bienestar, al menos tal y como había sido entendido en décadas anteriores (G. Esping-Ardensen, 1990, 1999), etc., constituyen, desde hace ya veinte años, áreas de gran relevancia para la investigación social. 
Las ciudades, en particular aquellas consideradas como "ciudades globales»" ${ }^{2}$, juegan un papel fundamental en el nuevo escenario emergente, generando unos modos de crecimiento que parecerían haberse hecho parcialmente autónomos, incluso respecto a la dinámica de las respectivas economías nacionales. A pesar de todo, los estados nacionales mantienen un papel importante, en la medida en que favorecen el reordenamiento de los procesos de acumulación capitalista dirigidos por el sector financiero y sustentados en la posibilidad, tecnológicamente factible, de la desconcentración territorial de la producción a escala global. En este proceso, los bloques comerciales y los «corredores logísticos», a través de los cuales el flujo del capital ha ido moldeando un nuevo paisaje global, se han reconfigurado. Así, vastos territorios del planeta y sus poblaciones han quedado rotundamente excluidos.

En este contexto, otros grandes centros urbanos, como México, San Pablo y Buenos Aires en América Latina, también juegan un papel estratégico en el establecimiento de nuevas inserciones globales diferenciadas de sus respectivos territorios nacionales, aunque en este caso cada vez más contundentemente subordinadas a estrategias geopolíticas y comerciales exógenas. En América del Sur, el corredor logístico latinoamericano que une Belo Horizonte con Santiago de Chile, involucrando en Brasil, además de a Belo Horizonte, a las ciudades de San Pablo y Porto Alegre (en conjunto, el territorio donde se genera el $40 \%$ del PIB del país); en Argentina, a Rosario, Córdoba, Buenos Aires y Mendoza (más del $60 \%$ del PIB argentino), y en Chile, la región metropolitana de Santiago y Valparaíso, eje que constituye el núcleo central de su dinámica económica (IDEP, 2000).

\section{Pobreza urbana, exclusión social y ciudad}

Dentro del corpus teórico de las Ciencias Sociales, el fenómeno de la pobreza urbana ocupa un lugar central. En efecto, desde los años ochenta aparecen cada vez con mayor nitidez signos de fragmentación y polarización social con características específicas respecto a etapas anteriores, de modo que es posible referirse a la nueva pobreza urbana (E. Mingione, 1993). Los grupos de jóvenes, las familias monoparentales, los ancianos de escasos recursos, los parados de larga duración, los inmigrantes, etc., constituyen el universo que la conforma.

El propio concepto de pobreza no es utilizado en el mismo sentido en todos los países. H. Silver (1996) establece la relación existente entre los diferentes discursos sobre la pobre-

2 Por ejemplo, Nueva York, Londres y Tokio, que concentran el comando de las principales decisiones que rigen la dinámica de la economía mundial y un conjunto de servicios avanzados que constituyen los soportes materiales y tecnológicos de este proceso (S. Sassen, 1991). 
za y los contextos nacionales en los que éstos se producen. Así, mientras en Francia, y en general en Europa continental, el debate mayoritariamente se produce en torno al concepto de exclusión social, en el área sajona, Gran Bretaña y EE.UU., la discusión se vuelca, fundamentalmente, alrededor del concepto de underclass y la formación del gueto pobre. Incluso los mismos conceptos tienen significados diferentes, dependiendo del país en el que se utilicen. Siguiendo a Silver, el término exclusión social debe ser comprendido en el marco de la ideología de la República, donde el principio de solidaridad aparece como eje en torno al cual se articula la integración nacional. La exclusión vendría a significar la ruptura de dicho vínculo. Señala también el carácter abierto del término y, por tanto, la necesidad de seleccionar aquellos aspectos de la exclusión social que marcarán nuestro propio punto de vista. En el área sajona, el principio de solidaridad es sustituido por el de comunidad.

R. Castel (1997) se refiere a la exclusión social entendiéndola no como un estado, sino como un proceso, que como tal debe ser enmarcado históricamente ${ }^{3}$. La precariedad laboral, junto a la fragilidad en cuanto a los soportes relacionales, conforman una zona de vulnerabilidad en la que se ubicaría una parte importante de la población. Esta zona debe ser diferenciada de aquella otra propiamente de exclusión social, de gran marginalidad, no únicamente sustentada en la ausencia de ingresos, sino en su alejamiento de las redes básicas de integración social. Así, el objeto de atención no deben ser únicamente los grupos sociales ya excluidos, sino también aquellos susceptibles de serlo. Este tipo de enfoque, ampliamente compartido en sus líneas generales por otros autores, aporta una gran riqueza interpretativa al análisis de los cambios recientes en la estructura social. Por tanto, nos encontramos ante un concepto complejo, polisémico, no vinculado a una única variable explicativa. Por ejemplo, la relación entre la exclusión del acceso a un empleo de calidad y la pobreza no es un hecho inmediato. Otros factores, como la duración de las situaciones de desempleo, la edad, el sexo, la inclusión en hogares con distintas capacidades de apoyo o el alcance de las políticas sociales, deben ser tenidos en cuenta al abordar la caracterización de la pobreza y la exclusión. Como afirma E. Mingione (1996), el empobrecimiento severo camina desde la progresiva descualificación hasta la exclusión completa del mercado de trabajo formal. La vulnerabilidad se convierte en más extrema para las mujeres, puesto que ellas sufren especialmente la explotación del mercado desregulado de trabajo y el desmantelamiento de las ayudas públicas dirigidas a aliviar la carga sobre los hogares de determinadas tareas de reproducción social.

Profundizando en esta idea de vulnerabilidad, D. Benassi y E. Mingione (1996) distinguen entre Estado de Bienestar, circunscrito al ámbito estatal, y Sistema de Bienestar, referido

${ }^{3}$ El propio autor advierte sobre la utilización del término ya que, en sentido estricto, nadie puede estar completamente excluido de la realidad social. 
tanto a la esfera pública como a la privada y formado por el soporte que proporcionan la familia, el mercado y el estado. De este modo, señalan la importancia de los sistemas de protección social existentes en cada país a la hora de frenar la caída de grupos sociales desde áreas de inestabilidad o precariedad hacia ámbitos de marginalidad. La construcción social de la pobreza queda ligada así al sistema moderno de ciudadanía y al welfare mix, es decir, a la combinación de oportunidades y apoyos que garantizan a todos los ciudadanos la satisfacción de su supervivencia y el acceso a unas condiciones de vida aceptables de acuerdo a los parámetros de su comunidad de pertenencia.

En ciertos casos límite, la extrema labilidad que existe entre la franja de exclusión social y la zona de vulnerabilidad se manifiesta de un modo particularmente grave, dado que las situaciones «de caída sin red» se perfilan como horizonte no ya sólo factible, sino cercano, para franjas muy amplias de población. El análisis del sistema de bienestar deja entonces a la luz un panorama de profunda desestructuración, en el que incluso las redes familiares se ven muy severamente debilitadas ${ }^{4}$.

La relación dialéctica existente entre espacio y sociedad nos sitúa en una perspectiva dinámica desde la cual los fenómenos de exclusión social son a la vez productores y producidos en su relación espacial. En este sentido, E. Mingione (1996), aludiendo a W. J. Wilson y su interpretación sobre el gueto pobre, destaca cómo la concentración de ciertos grupos en entornos cerrados y muy marcados por características étnico-sociales se convierte en un factor potenciador de exclusión social, independientemente de la acumulación de privaciones que puedan padecer. Al respecto, L. Wacquant (2001) destaca cómo la que él denomina nueva marginalidad tiende a concentrarse en determinadas áreas, donde el acceso de personas no residentes se convierte en prácticamente imposible. Esta tendencia contrasta notablemente con la distribución espacial de la pobreza en los años del desarrollo industrial de postguerra, mucho más transversal, presente en los distritos obreros y, en general, sin alcanzar niveles tan extremos de segregación espacial. En la actualidad, «el barrio ya no representa un escudo contra las inseguridades y las presiones del mundo exterior, un paisaje familiar y reafirmante imbuido de significados y formas de mutualidad colectivos» (L. Wacquant, 2001: 179).

\footnotetext{
4 Así ocurriría, por ejemplo, en el caso de la actual crisis argentina. En los últimos veinticinco años la población del país ha pasado de 25 a 37 millones de habitantes, mientras que la cantidad de personas en situación de pobreza, estimada según la "línea de pobreza», ha ascendido desde 2 a 17 millones; 6 de cada 10 de estas personas provienen de grupos de ingresos medios (C. Lozano, 2002). En el mismo periodo, las políticas públicas de corte universalista fueron muy restringidas, incorporando como parte de la reestructuración estatal los conocidos criterios de «focalización». A la vez, la curva del desempleo creció de manera casi constante debido, fundamentalmente, al desmantelamiento del sector industrial, la reconfiguración del aparato estatal y las privatizaciones. La creación de nuevos servicios terciarios y la expansión del sector informal, que acompañaron esta etapa económica basada en el predominio del capital financiero, ciertamente no compensaron el proceso exacerbado de concentración del ingreso.
} 
De todos modos, L. Wacquant concluye que, hasta la fecha, en Europa Occidental no es posible referirse a la existencia de una guetificación barrial como la observada en Estados Unidos, es decir, con una formación socioespacial segmentada y paralela que cumpliría la doble finalidad de la explotación y el ostracismo de una determinada categoría étnico-racial. Así, todavía no existiría un gueto turco en Berlín o un gueto árabe en Marsella. La existencia en estas ciudades de áreas espaciales caracterizadas por estas afinidades étnicas no ha implicado hasta la fecha la conformación de guetos. En una línea semejante, E. Mingione (1996), al comparar los modelos de polarización de las ciudades norteamericanas y del norte de Italia, concluye que en el segundo caso la aparición del gueto es mucho menos probable, definitiva y no tan caracterizada por un cierre étnico-racial. Sin embargo, sí es cierto que en las ciudades septentrionales italianas, al igual que en otras de Europa Occidental, las minorías empobrecidas y los inmigrantes tienden a concentrarse en las ciudades grandes, tanto en zonas centrales como periféricas. Dichos grupos se enfrentan a una doble penalización: en el acceso a las oportunidades de trabajo y en el acceso a distintos servicios de bienestar como la salud, la educación y la información, sin duda factores centrales para su promoción social.

La complejidad de los procesos vinculados a la pobreza y la exclusión en la ciudad convierte en poco explicativa la referencia a la imagen de la ciudad dual. J. Mollenkopf y M. Castells (1991), dos de los autores que más han contribuido a extender la metáfora de la dualización, al estudiar Nueva York concluyen que lo único homogéneo en términos sociales y de actuación política es el «corazón económico»: mayoritariamente blanco, masculino y formado por propietarios, gerentes y profesionales. Fuera aparece una «variedad caleidoscópica» de grupos subordinados y periféricos a ese desarrollo en términos de inserción en el mercado laboral, ocupaciones, etnia, origen, género, formas de habitar, participación comunitaria o modalidades de vinculación en relación a las políticas públicas. El accionar de cada uno de estos grupos produce clivajes distintivos en las ciudades postindustriales.

Pero, tal y como señala P. Marcuse (1995), tras el aparente caos de la ciudad postmoderna, fragmentada y dividida, es posible vislumbrar ciertos órdenes. Cada fragmento aparece separado del otro, pero esto no significa que no existan relaciones de interdependencia entre ellos. Sin embargo, no resulta sencillo captar los vínculos que unen los fragmentos ya que toda una trama superficial los oscurece. Frente a la metáfora de la ciudad dual, P. Marcuse prefiere utilizar la de la ciudad segmentada (partitioned), distinguiendo cinco tipos de barrios $^{5}$ que constituyen unidades separadas pero interdependientes. Dentro de la ciudad residencial: la ciudad dominante (de las torres fortaleza, ocupadas por la cúspide de la je-

5 P. Marcuse toma como referencia empírica las ciudades norteamericanas. Seguramente, en otras ciudades, las cinco grandes categorías establecidas no resultarían completamente pertinentes. De todos modos, a pesar de que éstas no fuesen las mismas, la lógica subyacente permite establecer cierta similitud. 
rarquía económica, social y política); la ciudad de la "gentrification» (ocupada por grupos profesionales, gerentes y técnicos de alto nivel adquisitivo); la ciudad suburbana (sectores medios que habitan en viviendas unifamiliares, trabajadores cualificados y empleados públicos); la ciudad de los inquilinos (que incluye vivienda social y, en general, económica, ocupada por trabajadores de baja cualificación), y, por último, la ciudad abandonada (de los pobres, en su mayoría desempleados, incluyendo también los sin techo). Por otro lado, la ciudad de la actividad económica impondría sus propias líneas de segmentación, no necesariamente coincidentes con las esbozadas anteriormente. Los patrones de actividad económica definen áreas donde individuos muy heterogéneos en términos de clase, ocupación y estatus trabajan en proximidad (la ciudad del control, la ciudad de los servicios avanzados, la ciudad de la producción directa, la ciudad de los trabajos no calificados y la economía informal en pequeña escala y la ciudad residual). Los «muros» que limitan los distintos fragmentos de ambas ciudades (la residencial y la de la actividad económica) pueden ser tangibles o intangibles, materiales o simbólicos, y cumplen diversas funciones. A través de ellos pueden rastrearse los grupos sociales y los intereses concretos que los originan, sustentan y reconfiguran, constituyéndose en indicadores excelentes para indagar en la naturaleza de las relaciones sociales y de poder.

En la escala local (A. Madanipour, 1998), la «espacialización» de la exclusión social se produce a través de los mercados de la tierra y propiedad, junto con una tendencia hacia el diseño y el planeamiento orientada a regular y racionalizar la producción del espacio para la imposición de un orden concreto. Además, si se admite que la exclusión social viene marcada por una imposibilidad de acceder a..., la propia existencia de espacio público y privado indicaría la importancia que este último adquiere como factor de exclusión.

Profundizando en esta misma línea, otros autores han interpretado las tendencias a la gentrification de las áreas centrales de las ciudades, observadas con bastante generalidad a partir de los años ochenta del siglo xx, como una consecuencia de la profunda reorganización socioespacial que se está produciendo a escala global y, justamente, como la contracara de esa realidad del aumento de la nueva pobreza urbana.

El concepto de gentrification, utilizado por primera vez por la socióloga Ruth Glass en 1964, ha traspasado sus márgenes originarios para incluir bajo esta consideración no sólo la renovación residencial de áreas deterioradas y ocupadas por sectores de población de escasos recursos, sino también los nuevos desarrollos urbanos, como por ejemplo la recuperación de áreas industriales en desuso. En esta línea, los trabajos de N. Smith (1996) constituyen toda una referencia. Aunque su mayor producción se centra en la experiencia de las ciudades estadounidenses, también repara en el ámbito europeo y, así, París, Amsterdam o Berlín constituyen los escenarios sobre los que traza la geografía de dicho fenó- 
meno. El autor adopta una visión integradora y global en su análisis, donde tienen cabida tanto las interpretaciones basadas en la esfera del consumo como en la de la producción, perspectivas que funcionaron separadamente en muchos de los estudios sobre gentrification. N. Smith trata de contextualizar los procesos de gentrification en un esquema mundial de desarrollo desigual reforzado por el fenómeno de la globalización. Las mismas fuerzas que generaron la gentrification en la ciudad central son las que han ido transformando los suburbios. La re-centralización de oficinas, comercios, establecimientos de ocio, hoteles, etc., ha ido acompañada de una descentralización que ha profundizado la fragmentación espacial en los territorios donde se localizan las nuevas urbanizaciones.

El surgimiento de trabajos muy cualificados y de elevada remuneración en torno al mundo de las finanzas, propio de la ciudad postfordista, junto a la presencia creciente de profesionales liberales, artistas y creadores en general, han generado cambios importantes en los patrones de consumo de estos grupos, basados en la especialización y la significación a través de la utilización de elementos de distinción. S. Zukin $(1995,1998)$ señala cómo el capital simbólico, que representaba la cultura en épocas precedentes, se transformó en una estrategia comercial donde la cultura se convierte en objeto de consumo, produciendo fuertes transformaciones en la conformación del espacio urbano. En lo que la propia autora denomina modo de producción artístico, destaca, entre otras, la rehabilitación patrimonial como una estrategia económico-cultural orientada justamente a satisfacer esa demanda especializada de consumo, a la vez que señala la alianza establecida entre capital y cultura para orientar la atención hacia aquellos aspectos de la estrategia menos conflictivos, es decir, los culturales, desviando la mirada de esa otra faceta más espinosa: la económica6.

Estos fenómenos, que básicamente se concentran en las ciudades globales, tienen también su expresión en ciudades más pequeñas y de rango inferior respecto a los circuitos mundiales de la globalización. Ante la especialización creciente que van adquiriendo las ciudades en los ámbitos nacionales e internacionales en orden a ofrecerse como mercancía consumible, se impulsan diversas políticas urbanas que, lejos de orientar su actividad hacia la construcción de una ciudad más igualitaria y democrática, agotan sus recursos en la potenciación de proyectos de dudoso beneficio social (F. Díaz Orueta y M. L. Lourés, 2003).

\footnotetext{
6 P. Marcuse (1998), refiriéndose a la reconstrucción de Berlín como sede del gobierno alemán, destaca la existencia de un amplio foro de discusión acerca de los nuevos espacios que se están construyendo, al que concurren tanto figuras nacionales como internacionales para discutir sobre cuestiones referidas a las formas arquitectónicas, sin que esto signifique el cuestionamiento esencial, es decir, el hecho mismo de la construcción y sus repercusiones económicas y sociales. En definitiva, se puede discutir el cómo pero no el qué.

7 En ese sentido, la reconsideración del sentido otorgado a la práctica de la planificación urbana y el desarrollo de la planificación estratégica aplicada a las ciudades, se convierten en aspectos esenciales para comprender la actuación de las distintas administraciones y del sector privado como gestores del crecimiento urbano más reciente. Sobre la planificación estratégica: J. M. Fernández Güel (1997).
} 


\section{BUENOS AIRES Y SU INSERCIÓN EN EL CONTEXTO DE LA GLOBALIZACIÓN}

Más allá del carácter específico y particular de cada uno de los países que componen el mosaico latinoamericano, el contexto de globalización en el que se desarrolla la fase actual del capitalismo permite señalar, a grandes rasgos, algunas características compartidas por todos ellos. Desde principios de los años noventa y en concordancia con el proceso aperturista iniciado a mediados de los setenta, las grandes ciudades latinoamericanas han recibido un importante flujo de capitales e inversiones extranjeros directos. Las privatizaciones de bienes y servicios, junto a la desregulación de los mercados financieros, fueron elementos clave para sustentar este proceso. Es así como las grandes urbes latinoamericanas han visto reforzado su papel como centros neurálgicos de actividades financieras, de intercambio y asentamiento de empresas transnacionales, situándose, en la jerarquía de las ciudades globales, en un segundo circuito con relación a aquellas que ocupan el lugar de comando. En México, San Pablo o Buenos Aires, el impacto territorial de estos flujos de capital e inversiones directas se ha ido haciendo perceptible, materializándose en la aparición de nuevas geografías sociales. Simultáneamente, las ciudades intermedias experimentaron un crecimiento notable debido, en gran parte, al asentamiento de empresas destinadas a la exportación, cuya ubicación se corresponde con un modelo de implantación empresarial desterritorializado orientado, básicamente, a buscar la localización más ventajosa.

La reorganización del aparato estatal corrió pareja a los cambios económicos propiciados. La aplicación de las políticas de ajuste estructural, incluso en etapas de crecimiento económico, vino a ahondar procesos de empobrecimiento de la población carenciada, sumergiendo a amplios sectores sociales en un estado de vulnerabilidad permanente. El universo social constituido por la nueva pobreza urbana se nutrió, fundamentalmente, de las clases medias. Por su parte, aquellos que venían padeciendo la pobreza severa vieron rebajados sus mínimos de subsistencia hasta cotas inimaginables.

En un marco de creciente desigualdad, y frente a la reorganización socioespacial que tuvo lugar, con signos evidentes de fragmentación y segregación, los viejos y nuevos problemas urbanos adquirieron una gran magnitud, de modo tal que, en el contexto socioeconómico y político actual, las posibilidades de favorecer un proceso de urbanización equilibrado socialmente y respetuoso con el medio ambiente resultan muy limitadas (F. Díaz Orueta, 1997).

M. Lungo y S. Baires (2001: 6) identifican tres tipos de intervenciones urbanas que han contribuido de forma notable a marcar y profundizar el carácter segregador del nuevo espacio urbano: 
a) Los grandes proyectos de renovación urbana, asociados, generalmente, a la potenciación de procesos de gentrification. La recuperación de centros históricos cabría encuadrarla dentro de este tipo de proyectos.

b) La construcción de grandes infraestructuras viarias y centros comerciales, ligados con la relocalización de actividades productivas y servicios y con el comercio internacional e interregional, vinculado al proceso de globalización.

c) La creación de urbanizaciones cerradas en áreas de rentas altas y medio-altas. Estos complejos residenciales adquieren formas variadas dependiendo de la ciudad y/o país del que se trate, conectándose con las nuevas redes viarias urbanas.

Estas intervenciones, que constituyen proyectos centrales en las agendas urbanas de las grandes ciudades latinoamericanas, se constituyen como un entramado coherente de adaptación de las ciudades a este nuevo marco de globalización económica.

Buenos Aires metropolitana se encuentra entre las veinte ciudades más pobladas del mundo. Aunque ocupa menos del $1 \%$ del territorio del país, casi un tercio de la población argentina reside en ella. La superficie de Buenos Aires, la ciudad central del área metropolitana, se calcula en $200 \mathrm{~km}^{2}$, que albergaban en 2001 a 2.768 .772 residentes $^{8}$. Su población, que se mantenía estable desde 1947 alrededor de unos 3 millones de personas, experimentó en el último periodo intercensal (1991-2001) una pérdida de 196.631 habitantes. El área suburbana cuenta con $3.800 \mathrm{~km}^{2}$, divididos en 24 partidos, con una población total en 2001 de 8.684.953 personas, frente a los 7.952.624 de 1991. El conjunto de la aglomeración urbana de Buenos Aires ha pasado de 10.918.027 habitantes en 1991 a 11.453.725 en 2001.

Fundada por los españoles en el siglo xVI, se desarrolló como centro comercial y administrativo, funciones ligadas a su localización portuaria sobre la costa del Río de la Plata. En 1880, con la creación del «estado-nación», la ciudad fue designada capital de Argentina y declarada «distrito federal», administrado directamente por el gobierno nacional. En 1996, este estatuto político-institucional se modificó, al sancionarse su propia constitución y adquirir el carácter de "ciudad autónoma», lo que le permite contar con un intendente elegido directamente por su población.

En el contexto internacional y regional al que venimos haciendo referencia, Buenos Aires experimenta una transformación significativa cuyas raíces más inmediatas habría que bus-

8 Los datos provisionales del Censo de Población de 2001 en Argentina pueden consultarse en http://www.indec.mecon.ar. 
carlas en el proceso liberalizador iniciado a mediados de los setenta y que adquiere su mayor expresividad en la década de los noventa. En este periodo se ha reforzado la centralidad urbana de la ciudad de Buenos Aires, corazón de la región metropolitana, a través de un conjunto de proyectos de intervención que han supuesto la reurbanización de su ribera, de norte a sur, la ampliación de su área central ${ }^{9}$ y el desarrollo de un sistema de autopistas que facilita la comunicación entre la city y los barrios cerrados que emergen en la zona suburbana. Proceso este último que $\mathrm{H}$. Torres (2001), al referirse al carácter de la urbanización residencial de los noventa, calificó como de «suburbanización de las élites».

En el despliegue de estos procesos, la política urbana ha jugado un papel fundamental. Como indican $\mathrm{H}$. Herzer y otros (1999), las acciones gubernamentales de nivel nacional y local han sido gestoras de las condiciones necesarias, aunque no suficientes, para el surgimiento de transformaciones económicas, sociales y ambientales en áreas degradadas o recalificables de la ciudad. El caso de Buenos Aires es ilustrativo al respecto. El gobierno nacional ha incidido significativamente con la puesta a disposición, para su valorización, a muy bajos costos, de 170 hectáreas de tierras fiscales nacionales, a través de la creación de la «Corporación Antiguo Puerto Madero» ${ }^{10}$, posibilitando la expansión del área central, cuyo suelo disponible se encontraba agotado. En consonancia, el gobierno de la ciudad ha invertido en obras de infraestructura, fundamentalmente la realización de obras de defensa costera destinadas a mitigar las inundaciones en el barrio de La Boca, de localización continua sobre la ribera hacia el sur respecto Puerto Madero, y otras reorientaciones de la inversión pública ${ }^{11}$ realizadas bajo la ilustrativa consigna del primer gobierno de la ciudad elegido en forma directa, en $1996^{12}$, de «incorporar el sur al norte»13. Otras medidas que integraron el proceso fueron las modificaciones en el Código de Edificaciones y de Planeamiento Urbano, el diseño del Plan Urbano Ambiental y la creación de una Corporación del Sur ${ }^{14}$.

\footnotetext{
9 Mediante la incorporación de suelo urbano, a través del desarrollo y modificación funcional de áreas de infraestructura decaída o subutilizada, destinadas a nuevos usos comerciales, de servicios y habitacionales, que involucran a sectores de altos ingresos.
}

10 Creada el 12 de noviembre de 1989, e integrada a partes iguales por el estado nacional y el municipio. El 9 de septiembre de 1998 fue aprobado el barrio número 47 de la ciudad de Buenos Aires: Puerto Madero. Sobre la naturaleza de la intervención en Puerto Madero y su impacto sobre la ciudad de Buenos Aires: F. Díaz Orueta y M. L. Lourés (2003).

11 La asignación presupuestaria proyectada preveía que por cada 100 dólares invertidos en el norte, se dirigieran 130 a la zona sur, destinados a programas como «Buenos Aires y el Río» (obras de mejoramiento de la Costanera Sur), estudios para la realización de una futura línea del ferrocarril subterráneo, etc.

12 El primer jefe de gobierno de la ciudad de Buenos Aires fue Fernando de la Rúa, perteneciente al Partido Radical.

13 El sur es asociado a cierta preponderancia en la presencia de sectores de menores ingresos y a suelo urbano disponible debido a su menor densidad constructiva.

14 El impacto de la crisis actual ha implicado un impasse en este proceso. Únicamente se mantienen con un nivel de actividad significativo las obras de la segunda fase de Puerto Madero. 
Este conjunto de proyectos de recualificación urbana ha supuesto también transformaciones importantes en los barrios adyacentes a Puerto Madero. El barrio de San Telmo, que se identifica con el centro histórico de la ciudad, ha sido objeto de un encuadre normativo de protección patrimonial ${ }^{15}$, sucesivamente flexibilizado y permeable a la expansión de usos ligados a la city, pero tendiendo a recortar o preservar un «corazón histórico turístico» restringido a unas pocas manzanas, que han sido progresivamente rehabilitadas, junto con la aplicación de programas de corte «cosmético» como la recuperación de la iluminación y calzadas históricas o la ampliación de un circuito peatonal turístico que lo vincula con el barrio de La Boca ${ }^{16}$.

Una intervención semejante, y aún más pronunciada, se ha desarrollado en el barrio de tradición obrera y portuaria de La Boca, en torno al área de «Caminito»17. En su conjunto, estos proyectos no se acompañan por políticas dirigidas a mejorar las condiciones de hábitat $^{18}$ de los sectores sociales residentes en las zonas impactadas por los cambios, en particular los de menores ingresos.

De este modo, se perfila un proceso que pareciera acentuar la fragmentación socioterritorial, al vincular entre sí el conjunto de operaciones señaladas, integrándolas en un circuito único y a su vez marcando un carácter netamente diferenciado de sus entornos barriales próximos.

\section{DOS BARRIOS DIFERENCIADOS PARA UN CIRCUITO TURÍSTICO CULTURAL: SAN TELMO Y LA BOCA}

\section{San Telmo}

San Telmo es el barrio de la zona sur más próximo a los centros político y administrativo nacional y de la ciudad, localizado a 700 metros de la Plaza de Mayo, su centro político, y a

15 El 25 de julio de 1979 el gobierno militar dictó la «Ordenanza de Preservación del distrito U24», compuesto por 144 manzanas. En 1982 el área fue reducida a la mitad. En 1991, y al objeto de flexibilizar aún más la normativa, se sancionó la ordenanza que regula el funcionamiento de las Áreas de Preservación Histórica (APH), estableciéndose un catálogo con diversos niveles de protección para cada edificio. Para un estudio detallado de este proceso, véase M. L. Lourés (1997).

16 Integrando la Plaza Dorrego y las calles Defensa y Balcarce.

17 La calle Caminito, ubicada en el corazón de La Boca y de fuertes resonancias artísticas, representa una versión concentrada de los mitos pasados sobre el barrio.

18 Hábitat entendido en su concepción integral: vivienda, trabajo, salud, educación, cultura, en sus aspectos materiales, simbólicos y fundamentalmente políticos, que hacen a la constitución de la identidad y de la ciudadanía. En este sentido, el derecho a la ciudad, como reivindicación de un derecho que se ejerce colectivamente, se esboza en algunos ámbitos globales (II Foro Social Mundial de Porto Alegre, 2002) como la respuesta política a las consecuencias de la globalización y contracara de la fragmentación socioterritorial y los procesos de exclusión en las ciudades (C. Rodríguez, 2002). 
unos 1.000 metros de la city, su centro financiero. Cuenta con buenos soportes de servicios e infraestructura urbana y su estado de conservación, aun presentando lugares deteriorados, es muy superior a otros barrios linderos del sur.

Es un barrio pequeño, de aproximadamente 130 hectáreas, cargado de simbolismo, como lugar «mítico de la fundación» donde se localizaron, fundamentalmente durante el siglo XVIII y hasta la epidemia de fiebre amarilla de 1875, sucesivas generaciones de familias «patricias» de la sociedad porteña. Conserva el carácter de ciertos «hitos», como la resistencia a las invasiones inglesas, desplegada en torno al eje de la calle Defensa, o una cierta memoria del periodo independentista (la casa de French, uno de los próceres de la revolución, se sitúa frente a la Plaza Dorrego).

Tras la epidemia de fiebre amarilla, la población del barrio se modifica, los caserones patricios son reutilizados progresivamente por sus dueños, que se trasladan a otras zonas de la ciudad, como edificios de alquiler de piezas, los primeros conventillos, destinados a vivienda de los inmigrantes europeos que arriban en forma masiva hasta $1915^{19}$. San Telmo adquiere paulatinamente un carácter híbrido: influido por la «experiencia cosmopolita», la desaparición del zanjón (entubamiento de los arroyos terceros) y la aparición del tranvía acercan al barrio al norte, a las nuevas costumbres y hábitos de la incipiente city porteña, de sus ministerios y oficinas. La expansión del mundo del trabajo, de las fábricas, los ferrocarriles, el puerto y los depósitos, afectan a San Telmo desde el sur (barrios de La Boca y Barracas).

Los primeros indicios que pueden dar cuenta del proceso de renovación que parecía alentar la primera normativa de preservación de San Telmo de 1979 se remontan una década atrás: su impulso se llevó adelante desde ciertos actores locales, como el Museo de la Ciudad, sustentando microintervenciones barriales, como la feria de anticuarios, y la promoción de organizaciones sectoriales que acompañaron el proceso (anticuarios y comerciantes), pero sin inversión pública y con la oposición de los agentes inmobiliarios locales.

Desde mediados de la década de los ochenta, se iniciaron acciones privadas de rehabilitación de lofts y viejas casonas por parte de particulares de sectores medios. En diciembre de $1991^{20}$ se sanciona la Ordenanza que regula el funcionamiento de las Áreas de Preservación Histórica y el barrio de San Telmo queda comprendido dentro de la APH1. Paralelamente, se crea un programa especial (San Telmo-Monserrat) destinado a implementar in-

\footnotetext{
19 En la coyuntura de la primer guerra mundial y como parte de la política del estado argentino en su etapa de inserción internacional bajo el modelo agroexportador.
}

20 Durante la misma gestión local que participa de la creación de la Corporación Antiguo Puerto Madero. 
tervenciones físicas en el área. Estas medidas marcan una nueva etapa de impulso en las transformaciones barriales. Sin embargo, el cambio de gestiones locales, en el contexto de las transformaciones institucionales y políticas de la ciudad, interrumpe la continuidad de la acción pública en el proceso.

El contexto de apertura del crédito inmobiliario a mediados de los noventa posibilitó un cierto desarrollo en el accionar de agentes inmobiliarios en torno a las operaciones de rehabilitación, pero este proceso se interrumpió en 1997-98, más en sintonía con la dinámica económica general que con definiciones específicas de políticas. La dinámica ligada a usos turísticos sigue una continua expansión reforzada, más discursivamente que en lo presupuestario, por el Programa San Telmo-Monserrat, que es trasladado en 2001 desde la órbita de Planeamiento, donde había sido creado, a la de Cultura. Mientras estas transformaciones renuevan la composición social del barrio, en ciertos periodos se refuerza la presencia de sectores de bajos ingresos: los ocupantes de edificios, fenómeno muy extendido en el barrio, que llegan mayoritariamente durante los primeros años de la recuperación democrática, y los inquilinos de hoteles-pensión, que se expanden sobre finales de los noventa, como consecuencia de la política gubernamental de subsidios en hoteles para situaciones de «emergencia» (C. Rodríguez y V. Devalle, 2001).

Desde 1970 y hasta 1991, San Telmo reforzó su perfil social de barrio heterogéneo ${ }^{21}$. Por un lado, entre 1970 y 1980 experimentó una pérdida de población del 15,7\%, pasando de 36.200 a 30.500 habitantes, y del 7,9\% entre 1980 y 1991, alcanzándose los 28.100 habitantes en esta última fecha. En el periodo 1980-1991, esa disminución coincide con un rejuvenecimiento de la estructura de edades del barrio: el grupo entre 0 y 14 años se incrementa del 15,8 al 17,7\%, y el de 15 a 29 años, del 21,8 al 22,3\%. Estos datos evidencian la llegada al barrio de nueva población residente que, mayoritariamente, se ubica en las casas y edificios tomados y en los hoteles-pensión.

En 1991, y de acuerdo a los datos del Censo de Población, prácticamente todas las categorías socioeconómicas aparecían representadas en el barrio, configurando un cuadro caracterizado por una gran heterogeneidad social. El análisis del nivel educativo y del tipo de empleo refuerza esta imagen. Por ejemplo, en 1991 la tasa de actividad se situaba claramente por encima de la media de la ciudad $(61,7 \%$ frente a $57,7 \%)$, si bien los niveles de desempleo eran más elevados en San Telmo, especialmente entre las mujeres jóvenes (la tasa de desempleo entre las mujeres de 15 a 29 años era en San Telmo del 10,7\%, muy

\footnotetext{
21 La información correspondiente al Censo de 2001 desglosada por barrios no se encuentra disponible. Por ello, la información estadística utilizada en el artículo se detiene en el año 1991 en San Telmo. Para La Boca se cuenta con un estudio del gobierno de la ciudad de Buenos Aires (en adelante, GCBA), que ha permitido actualizar algo más los datos en algunos aspectos.
} 
por encima del $6,2 \%$ del conjunto de la ciudad). Al mismo tiempo, la población se ocupaba crecientemente en el sector servicios, verificándose un aumento del cuentapropismo y una disminución del empleo en el sector público (M. L. Lourés, 1997).

San Telmo sufrió en esos años una importante transformación espacial que se manifiesta muy elocuentemente en el plano de la vivienda. El barrio pierde progresivamente su carácter residencial. De las 19.300 viviendas ocupadas existentes en 1980 , se pasa a 18.000 en 1991. Se sustituye el uso residencial por otros como oficinas y aparcamientos en superficie. A la vez, se expanden las formas habitacionales precarias: en el periodo citado se reduce la población residente en viviendas en régimen de propiedad, pasando del 60,6 al $52,9 \%$, y aumentan un $6,2 \%$ los ocupantes precarios, convirtiéndose en 1991 en el 9,2\% de la población (M. L. Lourés, 1997).

En la actualidad, San Telmo mantiene un importante stock de inmuebles deteriorados, muchos de ellos destinados al uso de hoteles-pensión de propiedad privada ${ }^{22}$ y otros de propiedad pública, en su mayor parte ocupados por familias de bajos ingresos, hacia los cuales el GBCA no ha planteado políticas específicas. Sólo dos inmuebles de propiedad pública han sido regularizados ${ }^{23}$, a instancias de procesos reivindicativos y propositivos impulsados, durante toda la década, por una organización social de hábitat, el Movimiento de Ocupantes e Inquilinos (MOI). El resto van siendo desalojados en dosis homeopáticas o bien perduran en la indefinición.

En lo que respecta a las actuaciones del GCBA en políticas destinadas a lo habitacional, no existen programas centrados en los sectores de más bajos ingresos de San Telmo. Sí se ha implementado una línea de créditos para promover el establecimiento de jóvenes profesionales, que no ha tenido mayor desarrollo en el contexto de la crisis. Por el contrario, la operación más significativa, y aún inconclusa, ha sido la de propender a la relocalización de las más de 100 familias residentes en el Ex-Padelai, una ocupación de dimensiones excepcionales localizada en el edificio del antiguo Patronato de la Infancia, a unos 100 metros del corazón turístico barrial, desconociendo, o más bien desarticulando a lo largo de la década, un proceso previo por el cual la organización barrial surgida en el predio (una cooperativa de autogestión) había ganado el derecho a su regularización y rehabilitación. Bajo el mismo criterio se ha desocupado otro pequeño inmueble, considerado de «valor histórico», sustrayéndolo para un circuito solvente.

22 Entre los cuales hay más de 20 involucrados en el Programa de Emergencia Habitacional del GCBA. Por ellos se abonan cifras que duplican y hasta triplican los precios de mercado de esas mismas piezas.

23 Uno de propiedad nacional y otro municipal. 


\section{La Boca}

La Boca es un barrio de 330 hectáreas de superficie, óptimamente localizado en la zona sur de la ciudad: a diez minutos en autobús del centro de la ciudad, contiguo a San Telmo y Barracas, y muy bien conectado a su vez con el área sur del Gran Buenos Aires (Avellaneda, Quilmes, etc.). Presenta un entorno espacial con niveles significativos de degradación ambiental, dados por su carácter ribereño24; por ser un barrio bajo tradicionalmente inundable; por contener amplias zonas de depósitos, actualmente receptores de mercaderías de importación y un significativo nivel de tráfico pesado, que genera efectos perjudiciales, como vibraciones, en un parque habitacional antiguo y muy deteriorado. A todo ello se suma un serio problema estructural: todavía mantiene el sistema de red de desagües pluviales y cloacales original de la ciudad.

Desde los tiempos de la fundación de la ciudad, la boca del Riachuelo fue su puerto natural, y generaba una actividad legal así como otra ilegal, de contrabando de esclavos y mercaderías. Éstas se almacenaban en barracas que eran alquiladas a principios del siglo $\mathrm{xx}$ como depósitos o saladeros y curtiembres. Su urbanización se consolidó durante el primer periodo de metropolización de la ciudad (1869-1914), convirtiéndose en un primer anillo que rodeaba el casco de la ciudad.

Históricamente, La Boca se formó como barrio obrero de inmigrantes europeos, que allí habitaban en piezas alquiladas en los conventillos y, progresivamente, al mejorar su situación económica en un contexto nacional de crecimiento, se fueron mudando a otras zonas de la ciudad. Se caracteriza por sus viviendas de chapa, madera y colores, prototípicas de la inmigración genovesa, que otorgan un carácter peculiar al barrio, aunque en general se encuentran muy deterioradas. A comienzos del siglo xx, el $27 \%$ de sus 60.000 habitantes residía en los 331 conventillos del barrio.

El marcado crecimiento poblacional encontró su punto de inflexión en 1947, año en que, de acuerdo a los datos de los Censos de Población, comenzó un proceso de vaciamiento que se prolonga hasta la actualidad. Entre 1947 y 1991 el barrio perdió el 40\% de su población, pasando de 76.000 a 46.000 habitantes. A ello se suma su progresiva degradación físicoedilicia y ambiental, particularmente agudizada desde los años setenta, con la desactivación del puerto del Riachuelo y el progresivo cierre de gran parte de las industrias de la zona (navieras, textiles y de producción de alimentos), tendencia que nunca se revirtió.

En este contexto de pérdida de roles productivos y funcionales, despoblamiento y deterioro socioeconómico, el GCBA, a mediados de los años noventa, abrió la posibilidad de un pro-

${ }^{24}$ El Riachuelo se encuentra muy contaminado y genera malos olores. 
ceso de renovación, con el inicio de las obras de defensa costera contra las inundaciones por sudestadas y otros programas complementarios orientados a reforzar su rol turísticocultural. El programa preveía la eliminación definitiva de las inundaciones, obras de saneamiento, reordenamiento del sector turístico, obras peatonales, rezonificación del antepuerto, declaración de «urbanización parque» de los bajos de la autopista 9 de julio y rezonificación de otros sectores de suelo urbano, considerado «vacante» en el barrio (H. Herzer y otros, 1999).

El carácter histórico del barrio se vincula más con rasgos culturales, como la vida asociativa y política propia de su pasado obrero de comienzos del siglo $\mathrm{xx}$, que con la existencia de un patrimonio arquitectónico que, si bien es distintivo, se encuentra altamente deteriorado. En este sentido, La Boca nunca tuvo una normativa especial de protección (APH) hasta la actual gestión del GCBA, que definió como tal un área circunscrita a Caminito y Vuelta de Rocha, la ribera inmediata.

La Boca es un barrio habitado en buena medida por sectores de bajos ingresos. Datos de 1999 del GCBA marcan que el 50\% de su población vive en condiciones críticas de habitabilidad, lo que significa un importante deterioro de sus viviendas, además de niveles muy elevados de hacinamiento. El $30 \%$ de su población habita en viviendas con más de tres personas por cuarto. Para esta fecha, en el barrio vivían 39.670 personas, lo que representa una disminución del $13 \%$ respecto a 1991. Parte de estos habitantes son inmigrantes procedentes de países limítrofes, concretamente el 63\% de los extranjeros, que, para 1997, constituían el $11 \%$ de la población total del barrio ${ }^{25}$.

La precariedad habitacional es así un elemento distintivo de la caracterización barrial. A las situaciones de déficit evidenciadas en los inquilinatos, que representan el $60 \%$ de las viviendas populares del barrio, y las casas ocupadas, se suma la rápida densificación durante los últimos años de una villa-miseria asentada en sus márgenes.

Desde 1996, a partir de situaciones de desalojos en inquilinatos, se inició un proceso de movilización barrial que trata de evitar la expulsión de las familias. En una primera etapa, la Iglesia jugó un papel relevante en el desarrollo de este proceso reivindicativo, del cual se van desprendiendo distintas organizaciones, conformando un mosaico fragmentado que se ocupa de reivindicar distintas necesidades sentidas: la vivienda y, en los últimos años, fundamentalmente, el trabajo y la alimentación.

En esa época el GCBA, desde su organismo responsable, decide dar continuidad a las obras en los 21 conventillos de su propiedad, que habían sido comprados durante la déca-

25 En revista Habitar, n. ${ }^{\circ}$ 2, abril-junio 2001, p. 6. 
da de los ochenta como parte de una intervención más ambiciosa de recuperación barrial que no había prosperado, más allá del reciclaje de cuatro edificios (el RECUP-BOCA) ${ }^{26}$. En 1997, la Comisión Municipal de la Vivienda creó la Operatoria Reconstrucción de Conventillos, sustituyendo por obra nueva otros cuatro edificios. También, y como consecuencia de la movilización de las familias, implementó la Operatoria 525/97, que generó una línea de créditos blandos mancomunables para que las familias adquirieran algunos de los viejos inmuebles. Sin embargo, estas medidas no configuraron una línea de intervención relevante dentro de la propuesta de renovación barrial.

\section{NUEVAS GEOGRAFÍAS INTRAURBANAS, SEGMENTACIÓN BARRIAL Y PROCESOS DE EXCLUSIÓN}

El conjunto de procesos ligados con la transformación urbana del circuito Puerto Madero-San Telmo y La Boca no ha supuesto la automática sustitución de los sectores sociales residentes en los dos barrios por otros de mayores ingresos. Hasta la fecha, en ambos barrios, los intentos de apropiación orientados a su conversión en un espacio destinado a diversos usos terciarios (ocio, turismo, oficinas, etc.) y a su conversión en hábitat de sectores sociales de elevados ingresos no han resultado completamente exitosos. Una parte importante de sus poblaciones se ve inmersa en procesos de exclusión social profundamente ligados a la crisis de naturaleza estructural que sacude el conjunto del país.

Pero para comprender la realidad de la exclusión social en San Telmo o en La Boca no basta con atender a estos elementos generales. Entre otros factores que marcan diferencias y nos reenvían a una lectura compleja y multidimensional, se reconocen:

a) Condiciones ambientales diferenciadas. La Boca presenta un cuadro mucho más deteriorado, persistiendo los efectos de la contaminación fluvial y de la circulación de tráfico pesado, y manteniendo un estado extendido de deterioro edilicio. Por contraposición, en San Telmo sólo aparecen ciertos «enclaves» de deterioro muy acusado, como por ejemplo el Ex-Padelai, edificio ocupado por más de 100 familias que resalta por su localización en el corazón turístico barrial.

\footnotetext{
26 EI RECUP-BOCA fue un programa impulsado en el barrio por la primera gestión democrática (1983) y podría haber supuesto un impulso inicial a su recualificación urbana. Partió de un diagnóstico integral a partir del cual se plantearon un conjunto de proyectos. Sin embargo, sólo se avanzó, parcialmente y con dificultades, en la compra de 21 conventillos para realizar vivienda pública de interés social destinada a sus habitantes, incorporando criterios de rehabilitación. En esta propuesta, la cuestión habitacional era un componente central de la agenda de la recualificación, pero no existía un conjunto de actores sociales barriales que la sustentaran.
} 
b) En términos del precio de la vivienda, La Boca (500 dólares el metro cuadrado construido), a diferencia de San Telmo (700-800 dólares el metro cuadrado construido) ${ }^{27}$, presenta valores inmobiliarios sensiblemente inferiores a la media de la ciudad (1.000 dólares).

c) Heterogeneidad social muy pronunciada en San Telmo, con una población entremezclada de sectores de medios y de bajos ingresos y atravesada por la presencia de los usos del «centro» en el barrio. En La Boca el implante turístico pareciera constituir un espacio de más acentuada segmentación intrabarrial, respecto de un entorno socialmente más homogéneo.

d) En cuanto a la precariedad habitacional, ambos barrios comparten la modalidad extendida de ocupación de edificios, preponderantemente de propiedad pública en San Telmo y de propiedad privada en La Boca, dada por la extendida presencia de inmuebles deteriorados y en estado de abandono. En La Boca predomina distintivamente el inquilinato (alquiler de piezas en inmuebles privados, que a veces devienen ocupaciones por falta de pago) y la creciente expansión de villas-miseria sobre el Riachuelo y bajos o linderos de autopistas. Las acciones de gobierno también refuerzan la reproducción de la precariedad habitacional, aunque de forma diferenciada en ambos barrios: en San Telmo se destaca la presencia extendida de grupos de bajos ingresos habitando en hoteles subsidiados para atender la «emergencia habitacional», que presentan importantes niveles de deterioro físico; en La Boca, por su parte, se ha desarrollado una operatoria de créditos blandos (la 525/97), que permitía la compra de viejos edificios, pero no necesariamente su rehabilitación, por parte de las familias de bajos ingresos ${ }^{28}$. Por tanto, se plantean diferencias en los modos de habitar, las trayectorias habitacionales, las características y expectativas de los grupos sociales, así como desde el punto de vista de la propiedad de los inmuebles, las estrategias de los propietarios y las inserciones barriales.

\footnotetext{
27 Valores en dólares norteamericanos anteriores al mes de diciembre de 2001. Tras la crisis, el mercado inmobiliario se encuentra estancado, por lo cual todavía no se dispone de valores ajustados a la situación actual.
}

28 Estos créditos se encuentran actualmente suspendidos. Como consecuencia de las experiencias llevadas adelante por organizaciones sociales de San Telmo (MOI) y La Boca (Mutual de Desalojados), se impulsó un proceso de reivindicación y debate en la legislatura y con organismos del ejecutivo, que implicó la formulación de una alternativa superadora, la Ley 341 , sancionada en 2001, de Autogestión y Emergencia Habitacional, con la definición de su respectiva operatoria. La misma alienta la autogestión, que implica la administración directa de los recursos por parte de la población, organizada en coooperativas, mutuales o asociaciones, e integra en el financiamiento todos los componentes del proceso productivo del hábitat: contempla la rehabilitación, la asistencia técnica, e incluye los aspectos de capacitación de recursos humanos y sistematización de los procesos. También define plazos de devolución y tipos de interés acordes a la situación de ingresos, incluidos niveles diferenciales de subsidios, más flexibles que la 525. No obstante, en el marco de la crisis nacional, y dado que el GCBA habría decidido desarticular el organismo responsable de la política habitacional (la CMV), conservando exclusivamente el programa destinado a la ejecución de viviendas de sectores de ingresos medios a través de licitaciones empresariales, la continuidad de esta operatoria se encuentra en riesgo. 
e) Tiempos y marcas diferenciados en las acciones de gobierno dirigidas a la recualificación urbana. Mientras que en San Telmo puede rastrearse un proceso de transformación desde comienzos de la década de los setenta, en La Boca las marcas iniciales son más recientes ya que, aun cuando puedan rescatarse las intervenciones de la década de los ochenta, sin embargo, el cambio cualitativo se remonta a mediados de los años noventa, con la ejecución de las obras para impedir las inundaciones ${ }^{29}$.

Los factores mencionados contribuyen a dar como resultado dos modos de segmentación socioespacial a escala barrial, cuya descripción permite problematizar el análisis de la fragmentación social y urbana y los efectos de la progresiva consolidación de la operación que recorta el itinerario Puerto Madero-La Boca-San Telmo, en relación con el resto de sus entornos barriales. Modos diferenciados de segmentación socioespacial que aportan matices también diferenciales en la comprensión de los procesos de exclusión.

En San Telmo se reconocen líneas de segmentación creadas por la tendencia a la expansión del área central, que van determinando los usos de oficinas, la localización de algunas sedes de empresas, los aparcamientos en superficie, la presencia de numerosos locales sindicales, institucionales y de servicios educativos universitarios. Por otra parte, el ya mencionado reforzamiento del «corazón turístico», usos comerciales y de servicios que recortan el entorno de Plaza Dorrego, los ejes de las calles Defensa y Balcarce y se expanden sobre la primera en la pretensión de unificar el circuito turístico. Un recorrido que conecta, como se dijo, con el barrio de La Boca. Entre esos usos que se expanden configurando los lineamientos de «la ciudad de la actividad económica», se localizan los otros usos residenciales de población socialmente muy heterogénea: desde las casonas recicladas de sectores de ingresos medios y medio-altos, edificios de apartamentos, lofts, hoteles pensión y casas tomadas habitadas por grupos de bajos ingresos.

La centralidad del barrio y, como parte de esos espacios intersticiales de la ciudad residencial, la posibilidad de la ocupación de viviendas y edificios vacíos, en la mayor parte de las ocasiones de titularidad pública, aportan una especificidad al área que obliga a realizar matices importantes en el estudio de la exclusión y de su componente espacial. En las casas tomadas, la precariedad habitacional, desde el punto de vista físico, puede resultar equiparable a la encontrada en las villas-miseria, tanto del centro como de la periferia de la ciudad. Sin embargo, su ubicación central suaviza la situación, al aumentar las posibilidades de empleo, acortar los tiempos y costes de transporte y permitir un acceso más amplio

29 Que supusieron una inversión pública superior a 100 millones de dólares, ejecutada con un préstamo del Banco Interamericano de Desarrollo (BID). 
a diversos servicios y equipamientos. Es aquí donde la fragilidad en la que se encuentran los sectores sociales más desprovistos encuentra un punto de soporte mínimo para mantenerse.

En La Boca, por el contrario, el circuito turístico cultural muy acotado contrasta con la muy extendida presencia de inquilinatos y viejas casas en estado de deterioro, así como con la expansión de nuevas villas-miseria altamente precarias y densificadas sobre la zona ribereña, que se presentan como la otra cara de «Caminito», la cara aún no reconocida en los mapas oficiales. Coexiste también la presencia extendida de depósitos portuarios, el cerrado hábitat de "Catalinas Sur» ${ }^{30}$ y los nuevos conjuntos de edificios en altura ubicados en los escasos terrenos vacantes de Casa Amarilla, construidos con financiación pública y destinados a futuros residentes de sectores medios.

\section{ENTRAMADOS DE ORGANIZACIONES SOCIALES, BARRIOS SEGMENTADOS Y CIUDADANÍAS RESTRINGIDAS}

La dimensión política es un aspecto de fundamental relevancia a la hora de reflexionar en torno a la dinámica de las grandes ciudades en el contexto de la globalización. La escala microbarrial posibilita indagar algunas cuestiones distintivas de las relaciones sociales en torno a los «muros» de la ciudad segmentada, otorgando otra arista a la comprensión de los procesos de exclusión y a la exclusión como proceso social. Esta arista se refiere a los modos en que los distintos habitantes del barrio, y en particular los actores locales, son incorporados o participan en las agendas políticas que acompañan los procesos de renovación y las transformaciones barriales.

En este sentido, en los barrios de La Boca y San Telmo es posible identificar, en primer lugar, dos universos de organizaciones en barrios contiguos territorialmente, que no han mantenido, en términos generales, vinculaciones significativas entre sí. En segundo lugar, dentro de los barrios aparecen redes sociales que presentan percepciones diferenciadas y, en algunos casos, contradictorias sobre los procesos de cambio barrial. Se estructuran así nodos de conflictos que hacen a las cuestiones básicas del derecho a la ciudad en relación con el impacto de los procesos de recualificación y que, salvo en situaciones muy excepcionales, prácticamente no encuentran canales de expresión política o la posibilidad de constituirse política e identitariamente como espacios de antagonismo.

30 Conjunto de 2.200 viviendas en 32 torres, construido por la Comisión Municipal de la Vivienda (CMV) en 1965 y habitado por sectores medios y profesionales liberales. 
En el barrio de San Telmo, los dirigentes de diversas organizaciones perciben claramente la contraposición entre las obras de rehabilitación arquitectónica y de mejoramiento del entorno barrial circunscritas al circuito turístico en contraposición a la ausencia de obras en el resto del barrio. Algunos, aquellos ligados en su accionar a los sectores de menores ingresos, al trabajo social comunitario y cultural desde una perspectiva popular, vinculan estos cambios a un proceso de expulsión que se prolonga por décadas, sin llegar a cristalizar en una política explícita. Ellos mismos se sienten excluidos del proceso de gestión de demandas, no valoran positivamente las intervenciones fragmentadas y destinadas únicamente al fomento de la actividad turística. Al mismo tiempo, tienen una visión reactiva de la pobreza, como contracara negativa de los aspectos positivos del barrio. No obstante, no es posible identificar en forma continua y orgánica una acción colectiva que identifique intereses comunes. Como contrapartida, las organizaciones que representan actores económicos locales (comerciantes, anticuarios) son quienes enfatizan la preservación de los aspectos más tradicionales del barrio, se sienten relativamente convocadas como interlocutores del GCBA y tipifican la pobreza asociada a la ilegalidad, la marginalidad, la inmigración ilegal y las drogas. En unos y otros, resulta sintomática la imposibilidad de comprender la exclusión como un proceso social. Antes que nada, la interpretan como un statu quo (C. Rodríguez y V. Devalle, 2001).

En ese sentido, un hito de ruptura en esta percepción se vincula con las experiencias de regularización dominial a través del desarrollo del cooperativismo autogestionario, impulsadas por una organización social de vivienda (el Movimiento de Ocupantes e Inquilinos, MOI) que enuncia públicamente otra concepción de la apropiación del espacio urbano, debatiendo la idea de «desarrollo local» y la posibilidad de otra recualificación urbana, e impulsando propuestas de gestión asociada público-privada y la participación directa de las instituciones barriales en la definición de usos y espacios públicos. Sin embargo, la perspectiva del MOI no ha sido asumida localmente por un espectro amplio de la vida asociativa que permita amplificar su voz y signar más profundamente la agenda política barrial.

Al mismo tiempo, el fenómeno creciente del turismo y el conjunto de actividades asociadas al mismo parecieran ir construyendo una polarización, aunque no un debate, entre aquellos grupos cuya estructura apunta a la rentabilidad económica y consideran el fomento turístico como una de las palestras de reconocimiento y valorización de San Telmo, y aquellos otros que lo visualizan en términos conflictivos, como conciencia de los riesgos que puede suponer el potencial proceso de gentrification. De este modo, «lo turístico» resulta el espejo más claro del conflicto de intereses de la población de San Telmo, el lugar de las proyecciones que los habitantes hacen del barrio, donde el proceso de recualificación urbana ocurre en presente, profundizando una escenografía vaciada de sus relaciones sociales distintivas, que constituyen el corazón viviente de ese patrimonio: la diversidad de su gente. 
Se dibuja así un escenario donde la fragmentación territorial tiene como correlato la fragmentación de las demandas y la dilución de la posibilidad de articulaciones políticas, a escala local. A tal punto que a finales de 2001 , al desarrollarse el proceso de asambleas barriales que surge de la crisis nacional en todas las ciudades más pobladas del territorio argentino, en San Telmo se constituyen tres asambleas, que se corresponden con distintas presencias, ya sean partidarias o de las organizaciones definidas como preocupadas por el trabajo social y cultural, reforzando así todavía más las tendencias a la fragmentación.

En el barrio de La Boca, el sentido histórico del patrimonio cultural y la identidad del barrio también se ha convertido en un eje de debate entre los líderes de las organizaciones tradicionales del barrio. El debate gira entre quienes pretenden conservar un supuesto pasado original y quienes aspiran a adaptarlo y recrearlo a la luz de una lógica orientada a la atracción de inversiones privadas. Planteada así la discusión, la situación de la población de menores ingresos aparece desvinculada o ajena al sentido de la historia colectiva que se recrea. Organizaciones tradicionales «progresistas» perciben los cambios como una mejora de la calidad de vida para los vecinos, mientras las «preservacionistas» consideran que los beneficios no son para los habitantes del barrio, entre los cuales identifican a parte de los que tienen los niveles de pobreza más alta de la ciudad. Sin embargo, ambos grupos coinciden en la demarcación de "otros" vecinos (nuevos inmigrantes, delincuentes, ocupantes, caracterizados como «los que no quieren trabajar» en un contexto de niveles crecientes y muy acentuados de desempleo) y que debieran ser desplazados para que el barrio mejore. También existen en La Boca una serie de comedores, la organización más extendida numéricamente, ligados a las necesidades inmediatas de las familias. El trabajo con las urgencias de la población y la relación que se establece entre los líderes de comedores y los partidos políticos tradicionales, que no incluyen la recualificación urbana en la agenda de la ciudad, limitan el desarrollo de una postura crítica y aun la posibilidad de percibir el riesgo del desalojo que parecen percibir sus beneficiarios. Por último, también han surgido en La Boca algunas organizaciones reivindicativas, ocupadas en formular demandas de alimentación, empleo y vivienda para los sectores de bajos ingresos, que casi no establecen puentes con las anteriormente mencionadas. Estas organizaciones no logran expresar un sentido de identidad común, que las involucre nítidamente en la disputa simbólica y material de la apropiación de usos y espacios barriales por parte de las familias afectadas por las múltiples dimensiones de la exclusión, como legítimos habitantes con derecho a vivir en el barrio de La Boca y como parte activa de la historia, del presente y del futuro del barrio $(\mathrm{H}$. Herzer y otros, 2002).

La cuestión social establece una línea divisoria y una suerte de «división de tareas» entre las organizaciones. Comedores y organizaciones reivindicativas centran su actuación en los elevados niveles de pobreza, la falta de acceso a los equipamientos sociales y la super- 
vivencia alimentaria. La subordinación clientelar y la incapacidad para lograr articular proyectos de expresión política alternativa definen también la actuación de estos grupos. Por otro lado, las asociaciones tradicionales discuten el sentido del patrimonio histórico y la renovación, como titulares de una ciudadanía política restringida a unos pocos.

No obstante, aun en este contexto, en ambos barrios el trabajo de organizaciones sociales que batallan por el derecho a la ciudad, por la regularización en la titularidad de las viviendas y la implementación de políticas autogestionarias de hábitat a través de la conformación de cooperativas y, de manera más amplia, por el impulso de nuevas estrategias de desarrollo barrial, continúan introduciendo tendencias que pueden contrarrestar parcialmente los procesos de exclusión. No sólo porque supongan una mejora en las condiciones habitacionales, sino, fundamentalmente, porque reintroducen a estos sectores sociales como sujetos políticos a considerar. En este sentido, resultan significativos los esfuerzos por articular redes entre organizaciones de ambos barrios, que se caracterizan por reivindicar concepciones y prácticas autogestionarias en la defensa y la propuesta del derecho a la ciudad. Recientemente, en el marco de la agudización de la crisis nacional y de su enorme impacto en la ciudad, estas iniciativas se han visto reforzadas.

\section{BIBLIOGRAFÍA}

BENASSI, David, y MINGIONE, Enzo (1996): «Ristrutturazione economica e povertà urbana nei paesi industrializzati», Archivio di Studi Urbani e Regionali, n. ${ }^{\circ}$ 57, pp. 37-65.

BOYER, Robert (ed.) (1988): La flexibilité du travail en Europe, París: La Découverte.

CASTEL, Robert (1997): La metamorfosis de la cuestión social, Buenos Aires: Paidós.

CASTELLS, Manuel (1997-1998): La era de la información: economía, sociedad y cultura, Madrid: Alianza Universidad (3 tomos).

DÍAZ ORUETA, Fernando (1997): «La ciudad en América Latina: entre la globalización y la crisis», América Latina Hoy, n. ${ }^{\circ}$, pp. 5-13.

- (2001): Desequilibrios socio-territoriales en la Comunidad de Madrid en la etapa de reestructuración económica, Madrid: Universidad Complutense.

DÍAZ ORUETA, Fernando, y LOURÉS, María Luisa (2003): «La ciudad postfordista: economía cultural y recualificación urbana», Revista de Economía Crítica, ${ }^{\circ}{ }^{\circ}$, en prensa.

ESPING-ANDERSEN, Gösta (1990): The Three Worlds of Welfare Capitalism, Cambridge: Polity Press.

- (1999): Social foundations of postindustrial economies, Oxford: Oxford University Press.

FERNÁNDEZ GÜEL, J. M. (1997): Planificación estratégica de ciudades, Barcelona: Gustavo Gili.

HARVEY, David (1989): The condition of Posmodernity, Oxford: Blackwell. 
HALL, Peter (1988): Cities of tomorrow, Oxford: Blackwell.

HERZER, Hilda, y otros (1999): «Características de la población de un barrio en proceso de renovación: luces y sombras», Realidad Económica, n. ${ }^{\circ}$ 168, pp. 104-131.

- (2002): «El proceso de renovación urbana en La Boca: organizaciones barriales entre nuevos usos y viejos lugares», Historia Actual, próxima publicación.

IDEP (2000): Informe sobre la ciudad de Buenos Aires, Cuaderno n. ${ }^{\circ} 64$, Instituto de Estudios y Formación de la CTA.

LEBORGNE, A., y LIPIETZ, Alain (1994): «El posfordismo y su espacio», Realidad Económica, n. ${ }^{\circ}$ 122, pp. 117-143.

LOZANO, Claudio (2002): Análisis de la crisis argentina, Buenos Aires: IDEF-CTA. http://www.cta.rcc.com.ar.

LOURÉS, María Luisa (1997): Buenos Aires: centro histórico y crisis social, Alicante: ECU.

LUNGO, Mario, y BAIRES, Sonia (2001): «Socio-spatial Segregation and Urban Land Regulation in Latin American Cities», en Segregation in the City, Seminario organizado por el Lincoln Institute of Land Police (LILP), Cambidge, Massachusetts, 25 a 28 de julio.

MADANIPOUR, Ali (1998): «Social Exclusion and Space», en Ali Madanipour, Göran Cars y Judith Allen (eds.), Social Exclusion in European Cities: Processes, Experiences and Responses, London: Regional Policy and Development Series, 23.

MARCUSE, Peter (1995): «Not Chaos, but Walls: Postmodernism and the Partitioned City», en Sophie Watson y Katherine Gibson (eds.), Postmodern Cities and Spaces, Oxford: Blackwell, pp. 243-253.

- (1998): «Refections on Berlin: the meaning of construction and the construction of meaning», International Journal of Urban and Regional Research, vol. 22, n. ${ }^{\circ}$, pp. 331-338.

MINGIONE, Enzo (1993): Sociedades Fragmentadas, Madrid: Ministerio de Trabajo y Seguridad Social.

- (1996): «Urban Poverty in the Advanced Industrial World: Concepts, Analysis and Debates», en Enzo Mingione (ed.), Urban Poverty and the Underclass, Oxford: Blackwell, pp. 3-40.

MOLLENKOPF, J., y CASTELLS, Manuel (eds.) (1991): Dual City: Restructuring New York, New York: Rusell Sage Foundation.

RODRÍGUEZ, Carla (2002): «Producción social del hábitat: un esfuerzo transformador colectivo», en VV.AA., La otra ciudad posible, México: Coalición Internacional para el Hábitat.

RODRíGUEZ, Carla, y DEVALLE, Verónica (2001): «¿Exclusión social? Percepciones de organizaciones sociales e identificación de lugares de disputa en contextos de renovación urbana», en XIII Congreso de la Asociación Latinoamericana de Sociología, Antigua (Guatemala), noviembre.

RODRíGUEZ, Carla, y REDONDO, Aurora (2001): «Procesos de renovación urbana en el Área sur de la ciudad de Buenos Aires: San Telmo y La Boca», en XXIII International Congress-LASA 2001, Washington, DC.

SASSEN, Saskia (1991): The Global City, Oxford: Princeton University Press.

SILVER, Hilary (1996): «National Discourses of the New Urban Poverty», en Enzo Mingione (ed.), Urban Poverty and the Underclass, Oxford: Blackwell, pp. 105-138.

SMITH, Neil (1996): The new urban frontier. Gentrification and the revanchist city, London: Routledge.

TORRES, Horacio (2001): “Cambios socioterritoriales en Buenos Aires durante la década de 1990», EURE, vol. 27, n. ${ }^{\circ} 80$. 
WACQUANT, Loïc (2001): Parias urbanos. Marginalidad en la ciudad a comienzos del milenio, Buenos Aires: Manantial.

ZUKIN, Sharon (1995): The Cultures of Cities, Oxford: Blackwell.

- (1998): «Urban Lifestyles: Diversity and Standardisation in Spaces of Consumption», Urban Studies, vol. 15, n. ${ }^{\text {os }}$ 5-6, pp. 825-839.

The analysis of social exclusion in cities often leads in the direction of highly delimited areas where the most extreme situations of urban unrest are concentrated. In fact, when these are present, it is in those areas where the main intervention plans and programmes set in motion by the Administration come together. However, exclusion does not always present such a clear territorial expression. So then, the worsening of social fragmentation processes does not necessarily result in the formation of heavily segregated spaces. Analysed in this article is the recent evolution of the city of Buenos Aires and, more specifically, of two districts in its southern area, San Telmo and La Boca. In both cases, there are various projects for urban reassessment, which are linked to the development of cultural economy. However, the experiences studied, despite occurring simultaneously and within a context of structural crisis of the Argentinian economy, show up very important peluciarities. What we are talking about are two different socio-spacial formations, with different degrees of social articulation and, therefore, the construction of specific social responses to urban reassessment processes. 